\title{
Research on the Innovation of Urban Street Visualization Light Monitoring System based on GIS Technology
}

\author{
Yanrong Liu ${ }^{1, \text { a }}$ \\ ${ }^{1}$ Shaanxi Institute of International Trade \& Commerce, Xi'an, Shaanxi, 712046 \\ ${ }^{a}$ email
}

Keywords: Urban Street Light, Monitoring System, GIS Visualization

\begin{abstract}
The current street monitoring is more traditional way, backward management and inefficient. Now on the street lamp monitoring system research is the use of wireless network technology and GIS technology to achieve automatic monitoring of street lamps, but the lack of street lamp system logic structure analysis. According to the physical model of street lighting facilities, the paper analyzes the logic model, and designs the visualized streetlight monitoring system by $\mathrm{C} / \mathrm{S}$ mode. In the system, the user can quickly find the location of the street controller through the map, and bulk control of a range of lights off, thus breaking the current simple way to control the street, the real realization of the picture to see the lights, Lights, lights to view the service.
\end{abstract}

\section{Introduction}

Street lights are an important part of municipal facilities and street lamp monitoring system is a very important indicator of the level of urban modernization. The traditional street lamp monitoring system mainly relies on artificial or timed clock to control the switch, because each section carries out the independent control of the section, and there is an error between the timers, which causes the street light to open and close is not unified, and because of the lack of the corresponding detection means cannot monitor the working state, management backward, inefficient. Therefore, the use of new management methods and technology to achieve street lamp monitoring is the urgent need for street construction and management. At present, the research of street lamp management system mainly introduces wireless network technology and GIS technology to realize the automatic monitoring of street lamps. However, these studies only describe in detail the design method using wireless network and GIS technology, the lack of analysis of the topology of the street lamp, and the logic model of the streetlight system is not clearly given, which seriously affects the operation efficiency of the monitoring system. In order to solve these problems, the author uses the database technology to analyze the physical model of the streetlight system and establishes the relational structure model of the system. On the basis of the relationship between the street and light system, the system database is established and the visualization of the streetlight system is realized by GIS technology monitor.

\section{Present Situation Analysis of Visualization Technology of Street Lamps}

In recent years, with the development of the city, the installation of various types of street lighting facilities in the continuous improvement, and, urban lighting has evolved from a simple lighting function to beautify the urban environment, improve the image of the city an important part of investment. The larger the city, the more the amount of street lighting facilities, coupled with various types of supporting facilities, such as control boxes, cables, etc., together to build a large, complex urban street lighting system. With the completion of the construction of the entire street system, the monitoring and management of the various street lighting facilities in the whole system is also a huge project, especially to monitor the system when the street lamp facilities failure, should achieve rapid positioning, repair. How to ensure the city's street lamp system efficient and safe work, to achieve a variety of street lighting facilities visualization, information monitoring and management has become a hot research. 
Traditional street lamp management mainly take semi-automatic or manual way, although to a certain extent, to achieve real-time monitoring of street lamps, but fundamentally speaking, still did not achieve visual monitoring, the main information transmission instability and other three aspects:

With the continuous expansion of urban roads, street lamps and other equipment more and more, its function tends to be complicated, showing a massive and discrete data characteristics, which greatly increased the workload of street monitoring. As the monitoring unit lacks the scientific information transmission and the gathering plan, causes the street lamp terminal equipment to obtain the information hysteresis, the perception is not flexible, the data transmission speed is slow, the data wrong package and so on.

The number of street monitoring terminals and the geographical position are superior, showing the cross state, because of this, in the design of street monitoring system, usually ignore the visual display equipment running state of work, making it impossible to real-time monitoring of street lamp operation, Process, there is no control and monitoring functions of organic unity, which cannot directly through the system platform to master the working status of street lamps.

The traditional street lamp monitoring methods are mainly inductive control, photoelectric control, always control to semi-automatic and manual management, focusing on a section of the street lights to monitor, cannot be very good to achieve a single street lamp control, especially in the visual aspects lack of certain accuracy. Currently used in street lamp monitoring technology mainly Zig Bee technology and communication technology, but it is only used in voltage, signal wave stability of short-distance communication, the scope of application is small.

The number of facilities: a city in the size of the road or the district are basically equipped with street lighting facilities for lighting, coupled with the matching control box, cable, the total amount is calculated in million units, The amount of information is huge: each street lighting facilities have a certain amount of attribute information, such as geographical location, power, controlled information, monitoring information, due to the huge amount of its own facilities, so the amount of attribute data is also quite large. Spatial location characteristics: street lighting facilities are basically based on urban road network design and laying, so with the geographical space characteristics, especially in the monitoring system in the early warning information positioning, spatial features more obvious.

It is difficult to realize the spatial location of early warning information: Because the monitoring and early warning information has strong spatial position characteristics, the current monitoring system can only be used in the form of text difficult to achieve spatial analysis function: In order to rationalize the deployment of staff and equipment, usually need to analyze the entire street lamp system in the early warning of the fault (such as the address text description), that failure, cannot do warning failure of the spatial location of visualization and positioning; it is difficult to meet the requirements of information sharing: the establishment of street light monitoring and early warning information platform to achieve the street lights monitoring system of digital information construction, the development of street light monitoring and alarm system is the development trend of the entire industry, and the current street monitoring system of various data and data organization decentralized, management unstructured, difficult to achieve data sharing requirements.

\section{System Function and Its Design Research}

The streetlight monitoring subsystem is a visual display of the streetlight status, which monitors the state of the street lamp in real time. The subsystem is the main subsystem of the streetlight monitoring system. The subsystem also provides two functions of status query and data query. State of the query is divided into communication test, current query and mode query three functions. Communication test for testing the upper and lower computer communication, you can understand which the next bit machine can be successfully controlled, which under the machine communication problems cannot be remote control, so that maintenance personnel in a timely manner. The current query can query the current of each detection channel corresponding to each lower computer, and can truly reflect the working conditions of the load by the current value so that the operator can understand the field control situation. The mode query is a query on the mode of operation of the 
lower computer so that the operator can understand the working mode of the lower computer and avoid the misoperation of the lower computer when the host computer is restarted. The data query subsystem contains basic information queries and thematic queries. Basic information query refers to the user through the road name, interest name, such as a certain building, you can quickly find the location of the data on the map where the space and can view the surrounding data information. Special information query refers to the user on the electronic map users click on a street or a distribution box, you can view the basic information of the street or distribution box.

Street lighting system equipment is very complicated, according to its operating characteristics can be divided into simple equipment, complex equipment and lines. Simple equipment is with headlights, siege lights and high pole lights, complex equipment, box-type change, landing table box, distribution room and wiring wells. Line lights have power lines, street lights control lines, power lines are divided into the main power lines, power lines, power lines, including sub-supply lines and branch lines.

Street lights in the state will consume a lot of power, energy in the lack of modern society, how to achieve energy-saving emission reduction is a hot research. Therefore, the system considers how to minimize the consumption of energy in the case of sufficient pedestrian demand. Each circuit of the distribution box is not controlled by the geographical position of the continuous distribution of street lamps, but the control of the street lights separated by two lights, a street on both sides of a distribution box by the three circuits control. In addition, the system uses the control system at intervals of two lights, in general, every night from 17:30 to 19:30, street lights separated by two lights, that is, control the road on both sides of the distribution box circuit all the way in the "open" State; from 19:30 to the next day 1:30, at this time more pedestrians on the road, in order to fully meet the needs of pedestrians, all lights are in the "light" state; from 1:30 to 6:00 the next day, Two lights of the control program; daily 6:00 to 17:30, street lights are in the "light off" state.

MapX is MapInfo company to provide users with a powerful map analysis of the ActiveX control products. Because it is a Windows operating system based on the standard controls, which can support the vast majority of standard visual development environment such as C \#, Delphi, VisualC ++ and so on. Programmers in the development process can choose their own most familiar with the development language, easy to map function embedded in the application, and can be out of Maplnfo's software platform to run. The basic operation of the electronic map includes the zoom display of the map, the mouse to achieve the map of the zoom, zoom, roaming and other basic operations.

As the display scale grows, the map display layer increases accordingly, displaying the map content more and more rich and detailed. Data query, according to the query results, the map according to a certain scale to the query results, and make the query results highlighted highlight. The design of the street monitoring system using C / S mode, the client uses C \# as the development language, the use of VisualStudio 2008 platform for the second development of MapX. Realize the real-time monitoring of street lamps, visually display the working status of street lamps and distribution boxes. In addition, the system also provides users with a friendly man-machine interface to help users easily use the system. The server of the system uses SQLServer, according to the relationship between the street lamp system structure model, the establishment of street monitoring system database for the system storage and provide GIS data and street lamps, distribution boxes and other equipment data. In the GIS database side, the database through the spatial data engine SDE through the window of the street color (gray light "light off", green said "light"), you can clearly get the work of the various lights. This interface gives the physical structure of the distribution box model and you can directly through the operation of the distribution box to change the work of the street lights to achieve the visualization of street lights.

\section{Conclusion}

The system uses GIS and database technology to complete the street lamp monitoring system design, the system not only uses the relational database to organize data, but also provides a visual street light status detection interface. However, due to time, knowledge and technical reasons, the system 
there are some shortcomings. Function: the system module function is not very comprehensive, cannot edit the elements of the layer, the query only supports accurate query, does not support fuzzy query. Technical aspects: The system does not consider the actual use of lights and distribution boxes in the communication problems, in the future work should be introduced wireless transmission module, enhance the practicality of the system.

\section{Acknowledgements}

Project: Based on GIS visualization of street lamp monitoring system innovation research in Xianyang Project Number: 2017JM6111

Xianyang promotion of scientific and technological achievements

\section{References}

[1] Qian Dongjie. A smart street lamp management design [J]. Technology to get rich guide. 2013 (20)

[2] Wu Guicai, Zhao Yun, Yang Tong. Study on interconnection and communication of intelligent streetlight management system based on single lamp control [J]. Technology and Enterprise. 2014 (09)

[3] Shi Wenbo, Zhang Zhongyu. Design and implementation of street lamp management system in Chenggang [J] .New China Communications. 2016 (12)

[4] Zhu Xiaobo. Using GIS to improve street lamp management level [J]. Tianjin Electric Power Technology. 1996 (02)

[5] Zhao Zhigang, Yan Li Shuang. Application and Design of GPRS in Urban Street Management System [J]. Journal of Harbin Normal University (Natural Science Edition)

[6] Wan Jianhua, Tao Xiangxiang, Liu Guoqing. Design of Intelligent Management of Urban Street Light Based on WebGIS and GSM Short Message [J]. Surveying and Mapping Engineering. 2007 (03) 